\title{
Measurement of Terrain Height by a Space Bistatic Radar System P-band with Reference to Destructive Ionosphere Effect
}

\author{
Oleg V. Goryachkin, Ivan V. Maslov \\ Povolzhskiy State University of Telecommunications and Informatics, Samara, Russia \\ oleg.goryachkin@gmail.com
}

\begin{abstract}
The possibility of using an interferometric bistatic acquisition in the P-band with a small spacecraft configuration - ground receiving point is considered. A theoretical study influence of the ionosphere on the accuracy of the reconstruction height of the terrain by a two-pass interferometer based on a P-band synthetic aperture radar (SAR) system and a ground receiving point is presented. The necessary statistical characteristics of random signal fluctuations are determined. An error of determining the height is presented depending on the geometry of the interferometer, the influence of the parameters of the ionosphere, additive noise, and spatial decorrelation of the radar images. Using the presented numerical dependences, it is possible to estimate the optimal range of values for the interferometric base and the error in determining the height for point and extended targets. When reflecting from extended targets, the error due to the spatial decorrelation of the reflected signals in one resolution element is additionally taken into account.
\end{abstract}

Keywords: bistatic sar, two-pass interferometry, ionosphere, P-band, small spacecraft 


\title{
ИЗМЕРЕНИЕ ВЫСОТЫ МЕСТНОСТИ КОСМИЧЕСКОЙ БИСТАТИЧЕСКОЙ РАДИОЛОКАЦИОННОЙ СИСТЕМОЙ Р-ДИАПАЗОНА С УЧЕТОМ ДЕСТРУКТИВНОГО ВЛИЯНИЯ ИОНОСФЕРЫ
}

\author{
О.В. Горячкин, И.В. Маслов
}

\author{
Поволжский государственный университет телекоммуникаций и информатики, \\ Самара, Россия \\ oleg.goryachkin@gmail.com
}

\begin{abstract}
Рассматривается возможность применения интерферометрической бистатической съемки в Р-диапазоне при конфигурации малый космический аппарат - наземный пункт приема. Представлено теоретическое исследование влияния ионосферы на точность восстановления высоты рельефа местности двухпроходным интерферометром построенном на базе радиолокационной системы с синтезированной апертурой (РСА) Р-диапазона и наземного пункта приема. Определены необходимые статистические характеристики случайных флуктуаций сигнала. Представлена ошибка определения высоты в зависимости от геометрии интерферометра, влияния параметров ионосферы, аддитивного шума, пространственной декорреляции радиолокационных изображений. По представленным численным зависимостям можно оценить оптимальный диапазон значений интерферометрической базы и ошибку определения высоты для точечных и протяженных целей. При отражении от протяженных целей дополнительно учитывается погрешность, обусловленная пространственной декорреляцией отраженных сигналов в одном элементе разрешения.
\end{abstract}

Ключевые слова: бистатический радиолокационный комплекс, двухпроходная интерферометрия, ионосфера, Р-диапазон, малый космический аппарат

\section{Введение}

При реализации интерферометрической съемки различают однопроходные (интерферометрическая база создается за один пролет РСА с двумя антеннами) и многопроходные (интерферометрическая база создается за два и более пролета РСА с одной антенной), однопозиционные и многопозиционные радиолокационные системы. Частным случаем многопозиционной системы является бистатическая конфигурация, когда передатчик и приемник разнесены в пространстве. Такая конфигурация позволяет существенно снизить массу и энергопотребление радиолокационного комплекса размещенного на борту малого космического аппарата. Данная схема построения бистатического радиолокационного комплекса c PCA (БиРЛК) Р-диапазона реализована на российском малом космическом аппарате "Аист2Д", выведенном на орбиту 28 апреля 2016 года [1]. Бортовая аппаратура БиРЛК представляет собой многорежимный импульсный передатчик, обеспечивающий широкую гамму стабильных 
сигналов на несущей частоте 435 МГц, в полосе до 6 (30) МГц с возможность перестройки от 1 до 30 МГц. Типы используемых зондирующих сигналов: последовательность прямоугольных импульсов, последовательность ЛЧМ-импульсов, последовательность фазоманипулированных сигналов, кодируемых М-последовательностью [1,2]. Бортовая передающая антенна представляет собой антенну Уда-Яги, поляризация - линейная, коэффициент усиления 5 дБ. Наземная приемная антенна «отраженного» канала представляет собой «стек» из двух антенн Уда-Яги, поляризация антенны - круговая, коэффициент усиления антенны отраженного канала 18-20 дБ, прямого - 9 дБ. При данных параметрах системы на расстоянии до 20 км в прямой видимости от приемной позиции обеспечивается чувствительность БиРСА $\left(\sigma_{0}\right)$ не хуже минус 20 дБ. Наземная стационарная аппаратура представляет собой двухканальный малошумящий приемник прямого усиления (до 110 дБ) с цифровой регистрацией 16-разрядных квадратурных компонент на частоте до 200 МГц и последующей цифровой обработкой сигналов. Импульсные сигналы бортового передатчика принимаются наземной аппаратурой (стационарного или мобильного исполнения) по двум каналам: «прямому» и «отраженному». Такая схема приема позволяет компенсировать фазовые искажения сигнала в ионосфере и обеспечивать когерентную обработку сигнала в РСА и получение пространственного разрешения до 5 м (при использовании полосы 30 МГц), в радиусе до 20 км [3,4].

\section{Двухпроходная интерферометрическая съёмка}

В отличие от работ [5,6], где проведен анализ влияния ионосферы для моностатической интерферометрической схемы съемки, в данной статье представлено исследование точности определения высоты местности двухпроходным бистатическим интерферометром Р-диапазона частот с наземной приемной частью. На рис. 1 схематично показана геометрия проведения эксперимента при двухпроходной интерферометрической съёмке. Малый космический аппарат, на борту которого находится радиолокационный БиРЛК, последовательно занимает положения МКА1 и МКА2, разнесенные в пространстве на базу $B$.

На рисунке $S_{o n 1}(t, x, y), \quad S_{\text {on2 }}(t, x, y)$ - комплексный опорный (прямой) сигнал от КА, $S_{n p 1}(t), S_{n p 2}(t)$ - комплексный принятый (отраженный) сигнал от области интереса, $B$ - база между двумя положениями (или пролетами МКА), $\alpha$ - угол наклона базы, $\theta$ - угол визирования, $H_{1}, H_{2}$ - высота орбиты первого и второго МКА, $D_{1}, D_{2}$ - удаление от области интереса, $h$ искомая высота рельефа местности.

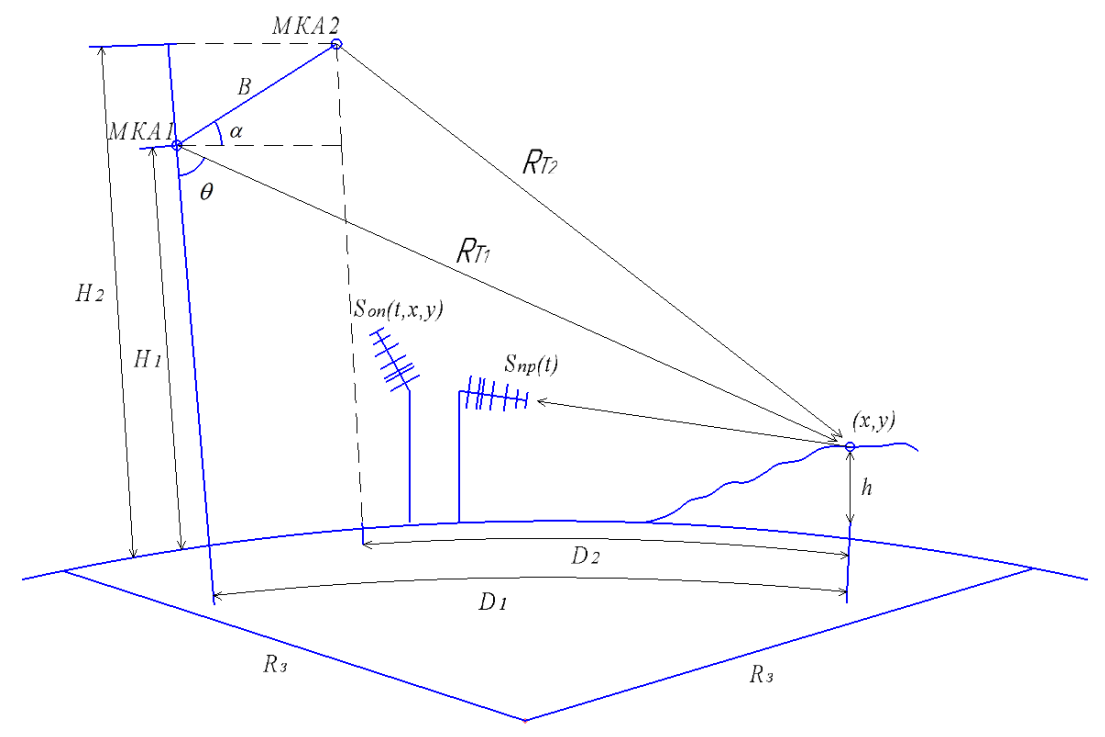

Рис.1. Схематичное изображение геометрии двухпроходной бистатической интерферометрической съемки 
При анализе погрешности интерферометрической РСА можно рассматривать отражение от стабильных отражателей с заданной ЭПР (точечная цель). В этом случае погрешность восстановления высоты цели определяется геометрией наблюдения, аддитивным шумом и влиянием атмосферы. При анализе погрешности интерферометрической РСА при отражении от поверхностно-распределенных целей (протяженная цель), необходимо дополнительно учитывать погрешность, обусловленную пространственной декорреляцией отраженных сигналов в элементе разрешения.

\section{Оценка влияния случайной составляющей ионосферной фазы на точность бистатического интерферометра}

Пусть $S_{0}(t)$ - последовательность зондирующих импульсов, излучаемая передатчиком РСА. Тогда сигнал, излученный 1-м МКА, отраженный от цели с координатами $(x, y, h)$ и принятый по прямой траектории

$$
\begin{gathered}
\dot{S}_{\text {omp } 1}(t)=\dot{\xi}_{1} \dot{S}_{0}\left(t-\tau_{12}(t, x, y, h)-\delta_{12}(t, x, y, h)\right) \\
\dot{S}_{n p 1}(t)=\dot{S}_{0}\left(t-\tau_{11}(t)-\delta_{11}(t)\right)
\end{gathered}
$$

где $\tau_{12}(t, x, y, h)$ и $\delta_{12}(t, x, y, h)$ - регулярная и случайная задержки сигнала на трассе МКА1-цельПП, $\tau_{11}(t)$ и $\delta_{11}(t)$ - регулярная и случайная задержки сигнала на трассе МКА1-ПП, $\dot{\xi}_{1}$ комплексный коэффициент отражения.

Сигнал точечной цели с координатами $\left(x_{0}, y_{0}, h\right)$ на 1-м РЛИ имеет вид

$$
\begin{aligned}
& \dot{I}_{1}(x, y)= \\
& =\dot{\xi}_{1} \int \dot{S}_{0}\left(t-\tau_{12}\left(t, x_{0}, y_{0}, h\right)-\delta_{12}\left(t, x_{0}, y_{0}, h\right)\right) S_{0}^{*}\left(t-\tau_{12}(t, x, y, 0)-\delta_{11}(t)\right) d t+\dot{N}_{1}(x, y) .
\end{aligned}
$$

где $\dot{N}_{1}(x, y)$ - аддитивный комплексный гауссовский шум для первого РЛИ, интегрирование производится на интервале синтеза апертуры $T_{s},\left[-T_{s} / 2, T_{s} / 2\right]$.

Используя равенство Рэлея для преобразования Фурье, разбивая интервал синтеза апертуры на интервалы, равные периоду повторения зондирующих импульсов $T_{r},\left[t_{k}-T_{r} / 2, t_{k}+T_{r} / 2\right]$, учитывая постоянство на этих интервалах задержки сигнала, получим

$$
\dot{I}_{1}(x, y)=\frac{\dot{\xi}_{1}}{2 \pi} \int\left|\dot{S}_{0}(j \omega)\right|^{2} \sum_{k} \exp \left(-j \omega\left(\Delta \tau_{12}\left(t_{k}, x, y\right)+\delta_{1}\left(t_{k}\right)\right)\right) d \omega+\dot{N}_{1}(x, y)
$$

где $\Delta \tau_{12}\left(t_{k}, x, y\right)=\tau_{12}\left(t_{k}, x_{0}, y_{0}, h\right)-\tau_{12}\left(t_{k}, x, y, 0\right) \quad$ - $\quad$ регулярная $\delta_{1}\left(t_{k}\right)=\delta_{12}\left(t_{k}, x_{0}, y_{0}, h\right)-\delta_{11}\left(t_{k}\right)$ - случайная. В этом выражении мы также полагаем, что регулярное искажение огибающей зондирующих импульсов на трассе распространения в ионосфере носит чисто фазовый характер и компенсируется при совместной обработке отраженного и прямого сигнала в ПП.

Пусть $\frac{1}{2 \pi} \int\left|\dot{S}_{0}(j \omega)\right|^{2} \exp (j \omega t) d \omega=\dot{g}_{0}(t)$, тогда

$$
\dot{I}_{1}(x, y)=\dot{\xi}_{1} \sum_{k} \exp \left(-j \omega_{0}\left(\Delta \tau_{12}\left(t_{k}, x, y\right)+\delta_{1}\left(t_{k}\right)\right)\right) g_{0}\left(\Delta \tau_{12}\left(t_{k}, x, y\right)+\delta_{1}\left(t_{k}\right)\right)+\dot{N}_{1}(x, y)
$$

Далее разложим $\tau_{12}\left(t, x_{0}, y_{0}, h\right)$ в ряд Маклорена по переменным $(t, h)$ и ограничимся следующими членами ряда

$$
\tau_{12}\left(t, x_{0}, y_{0}, h\right) \approx \tau_{12}\left(0, x_{0}, y_{0}, 0\right)+\tau_{12}^{\prime}\left(0, x_{0}, y_{0}, 0\right) h+\tau_{12}^{\prime}\left(0, x_{0}, y_{0}, 0\right) t+\frac{1}{2} \tau_{12}^{\prime \prime}\left(0, x_{0}, y_{0}, 0\right) t^{2}
$$


Тогда $\Delta \tau_{12}\left(t_{k}, x_{0}, y_{0}\right) \approx \tau_{12}^{\prime}\left(0, x_{0}, y_{0}, 0\right) h$ и $\Delta \tau_{22}\left(t_{k}, x_{0}, y_{0}\right) \approx \tau_{22}^{\prime}\left(0, x_{0}, y_{0}, 0\right) h$.

Положим далее, что разрешение по наклонной дальности таково, что миграция сигнала в канале дальности несущественна, т.е. $g_{0}\left(\Delta \tau_{12}\left(t_{k}, x_{0}, y_{0}\right)+\delta_{1}\left(t_{k}\right)\right) \approx 1$ для любого $\mathrm{k}$, тогда два РЛИ имеют вид

$$
\begin{aligned}
& \dot{I}_{1}\left(x_{0}, y_{0}\right)=\dot{\xi}_{1} \exp \left(-j \omega_{0} \tau_{12}^{\prime}\left(0, x_{0}, y_{0}, 0\right) h\right) \sum_{k} \exp \left(-j \omega_{0} \delta_{1}\left(t_{k}\right)\right)+\dot{N}_{1}\left(x_{0}, y_{0}\right), \\
& \dot{I}_{2}\left(x_{0}, y_{0}\right)=\dot{\xi}_{2} \exp \left(-j \omega_{0} \tau_{22}^{\prime}\left(0, x_{0}, y_{0}, 0\right) h\right) \sum_{k} \exp \left(-j \omega_{0} \delta_{2}\left(t_{k}\right)\right)+\dot{N}_{2}\left(x_{0}, y_{0}\right) .
\end{aligned}
$$

Интерферометрическая разность фаз в точке $\left(x_{0}, y_{0}\right)$ может быть найдена в виде

$$
\Delta \varphi=\arg \left[\dot{I}_{1}\left(x_{0}, y_{0}\right) I_{2}^{*}\left(x_{0}, y_{0}\right)\right]=\arg [\dot{z}]
$$

Подставляя (4) в (5), опуская $\left(x_{0}, y_{0}\right)$ получим

$$
\dot{z}=\dot{\xi}_{1} \xi_{2}^{*} \dot{I}_{01} I_{02}^{*}+\dot{\xi}_{1} \dot{I}_{01} N_{2}^{*}+\dot{N}_{1} \xi_{2}^{*} I_{02}^{*}+\dot{N}_{1} N_{2}^{*}
$$

где

$$
\begin{aligned}
& \dot{I}_{01}=\exp \left(-j \omega_{0} \tau_{12}^{\prime}\left(0, x_{0}, y_{0}, 0\right) h\right) \sum_{k} \exp \left(-j \omega_{0} \delta_{1}\left(t_{k}\right)\right), \\
& \dot{I}_{02}=\exp \left(-j \omega_{0} \tau_{22}^{\prime}\left(0, x_{0}, y_{0}, 0\right) h\right) \sum_{k} \exp \left(-j \omega_{0} \delta_{2}\left(t_{k}\right)\right) .
\end{aligned}
$$

В силу закона больших чисел $\dot{z}$ - случайный вектор, имеющий гауссовское распределение. Для оценки погрешности определения высоты найдем вероятностное распределение $\Delta \varphi$.

Математическое ожидание

$$
\mathbf{M}\{\dot{z}\}=m_{x}+j m_{y}=\exp \left(-j \omega_{0} \Delta \tau_{12}^{\prime} h\right) \mathbf{M}\left\{\dot{\xi}_{1} \xi_{2}^{*}\right\} \sum_{k} \sum_{l} e^{-\frac{1}{2}\left(D_{\delta_{1}}\left(t_{l}\right)-B_{\delta_{1} \delta_{2}}\left(t_{l}, t_{k}\right)+D_{\delta_{2}}\left(t_{k}\right)\right)}
$$

Где $\Delta \tau_{12}^{\prime}=\tau_{12}^{\prime}\left(0, x_{0}, y_{0}, 0\right)-\tau_{22}^{\prime}\left(0, x_{0}, y_{0}, 0\right), \quad D_{\delta_{1}}\left(t_{l}\right), \quad D_{\delta_{2}}\left(t_{k}\right)$ - дисперсии случайных величин $\omega_{0} \delta_{1}\left(t_{l}\right), \omega_{0} \delta_{2}\left(t_{k}\right), B_{\delta_{1} \delta_{2}}\left(t_{l}, t_{k}\right)$ - их ковариационная функция.

Дисперсия

$$
\begin{aligned}
& \mathbf{D}\{\dot{z}\}=\mathbf{M}\left\{\left|\dot{\xi}_{1}\right|^{2}\left|\dot{\xi}_{2}\right|^{2}\right\} \mathbf{M}\left\{\left|\dot{I}_{01}\right|^{2}\left|\dot{I}_{02}\right|^{2}\right\}-\left|\mathbf{M}\left\{\dot{\xi}_{1} \xi_{2}^{*}\right\}\right|^{2}\left|\mathbf{M}\left\{\dot{I}_{02} I_{01}^{*}\right\}\right|^{2}+\mathbf{D}\left\{\dot{N}_{1}\right\} \mathbf{D}\left\{\dot{N}_{2}\right\}+ \\
& +\mathbf{D}\left\{\dot{N}_{1}\right\} \mathbf{M}\left\{\left|\dot{\xi}_{2}\right|^{2}\right\} \mathbf{M}\left\{\left|\dot{I}_{02}\right|^{2}\right\}+\mathbf{D}\left\{\dot{N}_{2}\right\} \mathbf{M}\left\{\left|\dot{\xi}_{1}\right|^{2}\right\} \mathbf{M}\left\{\left|\dot{I}_{01}\right|^{2}\right\} .
\end{aligned}
$$

Далее, необходимо найти коэффициент взаимной корреляции реальной и мнимой части

$$
\begin{array}{r}
\mathbf{M}\{(\operatorname{Re}(\dot{z})-\mathbf{M}\{\operatorname{Re}(\dot{z})\})(\operatorname{Im}(\dot{z})-\mathbf{M}\{\operatorname{Im}(\dot{z})\})\}=\frac{1}{2}(\operatorname{Im}[(\mathbf{M}\{\dot{z} \dot{z}\}-\mathbf{M}\{\dot{z}\} \mathbf{M}\{\dot{z}\})]), \\
\mathbf{M}\{\dot{z} \dot{z}\}-\mathbf{M}\{\dot{z}\} \mathbf{M}\{\dot{z}\}=\mathbf{M}\left\{\left(\dot{\xi}_{1}\right)^{2}\left(\xi_{2}^{*}\right)^{2}\right\} \mathbf{M}\left\{\left(\dot{I}_{01}\right)^{2}\left(I_{02}^{*}\right)^{2}\right\}-\left(\mathbf{M}\left\{\dot{\xi} \xi_{1}^{*}\right\}\right)^{2}\left(\mathbf{M}\left\{\dot{I}_{01} I_{02}^{*}\right\}\right)^{2}+ \\
+\mathbf{M}\left\{\left(\dot{N}_{1}\right)^{2}\right\} \mathbf{M}\left\{\left(N_{2}^{*}\right)^{2}\right\}+\mathbf{M}\left\{\left(\dot{N}_{1}\right)^{2}\right\} \mathbf{M}\left\{\left(\xi_{2}^{*}\right)^{2}\right\} \mathbf{M}\left\{\left(I_{02}^{*}\right)^{2}\right\}+\mathbf{M}\left\{\left(N_{2}^{*}\right)^{2}\right\} \mathbf{M}\left\{\left(\dot{\xi}_{1}\right)^{2}\right\} \mathbf{M}\left\{\left(\dot{I}_{01}\right)^{2}\right\} .
\end{array}
$$

Если реальная и мнимая компоненты шумов в каналах независимы и имеют одинаковые дисперсии, то $\mathbf{M}\left\{\left(\dot{N}_{1}\right)^{2}\right\}=\mathbf{M}\left\{\left(\dot{N}_{2}\right)^{2}\right\}=0$, и

$$
\mathbf{M}\{\dot{z} \dot{z}\}-\mathbf{M}\{\dot{z}\} \mathbf{M}\{\dot{z}\}=\mathbf{M}\left\{\left(\dot{\xi}_{1}\right)^{2}\left(\xi_{2}^{*}\right)^{2}\right\} \mathbf{M}\left\{\left(\dot{I}_{01}\right)^{2}\left(I_{02}^{*}\right)^{2}\right\}-\left(\mathbf{M}\left\{\dot{\xi}_{1} \xi_{2}^{*}\right\}\right)^{2}\left(\mathbf{M}\left\{\dot{I}_{01} I_{02}^{*}\right\}\right)^{2}
$$

Дисперсия $\operatorname{Re}(\dot{z})$

$$
\sigma_{x}^{2}=\mathbf{M}\left\{(\operatorname{Re}(\dot{z})-\mathbf{M}\{\operatorname{Re}(\dot{z})\})^{2}\right\}=\frac{1}{2}(\mathbf{D}\{\dot{z}\}+\operatorname{Re}[(\mathbf{M}\{\dot{z} \dot{z}\}-\mathbf{M}\{\dot{z}\} \mathbf{M}\{\dot{z}\})])
$$


Дисперсия $\operatorname{Im}(\dot{z})$

$$
\sigma_{y}^{2}=\mathbf{M}\left\{(\operatorname{Im}(\dot{z})-\mathbf{M}\{\operatorname{Im}(\dot{z})\})^{2}\right\}=\frac{1}{2}(\mathbf{D}\{\dot{z}\}-\operatorname{Re}[(\mathbf{M}\{\dot{z} \dot{z}\}-\mathbf{M}\{\dot{z}\} \mathbf{M}\{\dot{z}\})])
$$

При анализе погрешности интерферометрической РСА можно рассматривать отражение от стабильных отражателей с заданной ЭПР (точечная цель). В этом случае погрешность восстановления высоты цели определяется геометрией наблюдения, шумом и влиянием атмосферы.

При отражении от поверхностно-распределенных целей (протяженная цель), дополнительно учитывают погрешность, обусловленную пространственной декорреляцией отраженных сигналов в одном элементе разрешения [6]. Для учета этой составляющей погрешности положим, что коэффициент отражения $\dot{\xi}_{1}$ формируется следующим образом

$$
\dot{\xi}_{1} \approx \int_{-\Delta x / 2}^{\Delta x / 2} \int_{-\Delta y / 2}^{\Delta y / 2} \dot{\eta}(x, y) \exp \left(-j \frac{2 \pi}{\lambda} y \sin \left(\theta_{1}\right)\right) d x d y
$$

где $\dot{\eta}(x, y)$ - коэффициент отражения диффузно рассеивающей поверхности, т.е. $\mathbf{M}\left\{\dot{\eta}(x, y) \eta^{*}\left(x^{\prime}, y^{\prime}\right)\right\}=\sigma_{0}^{2} \delta\left(x-x^{\prime}, y-y^{\prime}\right), \sigma_{0}$ - удельная ЭПР, - $x$ - разрешение по азимуту, $\Delta y-$ разрешение по поверхностной дальности, $\theta_{1}$ - угол падения.

Проведем далее численный анализ погрешности. Для круговой орбиты получим $\Delta \tau_{12}^{\prime}$ в виде

$$
\Delta \tau_{12}^{\prime}=\frac{1}{c}\left(\frac{R_{3}-\left(R_{3}+H_{1}\right) \cos \left(\frac{D_{1}}{R_{3}}\right)}{\sqrt{\left(R_{3}^{2} \sin ^{2}\left(\frac{D_{1}}{R_{3}}\right)+\left(R_{3}+H_{1}-R_{3} \cos \left(\frac{D_{1}}{R_{3}}\right)\right)^{2}\right)}}-\frac{R_{3}-\left(R_{3}+H_{2}\right) \cos \left(\frac{D_{2}}{R_{3}}\right)}{\sqrt{\left(R_{3}^{2} \sin ^{2}\left(\frac{D_{2}}{R_{3}}\right)+\left(R_{3}+H_{2}-R_{3} \cos \left(\frac{D_{2}}{R_{3}}\right)\right)^{2}\right)}}\right)
$$

Искомое распределение фазы в соответствии с [7] имеет вид:

$$
W_{4}(\varphi)=\frac{\sigma_{x} \sigma_{y} \exp \left(-\frac{m_{x}^{2}}{2 \sigma_{x}^{2}}-\frac{m_{y}^{2}}{2 \sigma_{y}^{2}}\right)}{2 \pi\left(\sigma_{y}^{2} \cos ^{2} \varphi+\sigma_{x}^{2} \sin ^{2} \varphi\right)}\left(1+k \sqrt{\pi} e^{k^{2}}[1+\Phi(\sqrt{2} k)]\right),-\pi \leq \phi \leq \pi
$$

Где $k=\frac{m_{x} \sigma_{y}^{2} \cos \phi+m_{y} \sigma_{x}^{2} \sin \phi}{\sigma_{x} \sigma_{y} \sqrt{\sigma_{y}^{2} \cos ^{2} \phi+\sigma_{x}^{2} \sin ^{2} \phi}}, \Phi(\sqrt{2} k)=\frac{2}{\sqrt{2 \pi}} \int_{0}^{\sqrt{2} k} e^{-\frac{t^{2}}{2}} d t-$ функция Крампа.

Учитывая представленную геометрию (рис. 1) запишем ошибку определения высоты

$$
\delta h=\sqrt{D\{\Delta \varphi\}} / \omega_{0}\left|\Delta \tau_{12}^{\prime}\right|
$$

где $D\{\Delta \varphi\}$ - дисперсия фазы, найденная по распределению (15).

В качестве исходных данных для расчета используем характеристики бистатического радиолокационного комплекса Р-диапазона, установленного на МКА «Аист-2Д», запущенного с космодрома "Восточный" в 2016 году, таблица 1. 
Табл. 1. Характеристики радиолокационного комплекса

\begin{tabular}{|l|l|}
\hline \multicolumn{1}{|c|}{ Параметр } & \multicolumn{1}{c|}{ Значение } \\
\hline Разрешение, азимут х дальность, м & 30 х30 \\
\hline Удаление КА от точки интереса, км & 500 \\
\hline Время синтеза, с & $0.8-2.2$ \\
\hline Ширина полосы частот, МГц & $6-30$ \\
\hline Отношение сигнал/шум для точечной цели, дБ & не хуже 14 \\
\hline Отношение сигнал/шум для распределенной цели, дБ & не хуже 16 \\
\hline Масштаб неоднородностей в ионосфере, м & $1000-10000$ \\
\hline Удельная ЭПР протяженной цели, дБ & -20 \\
\hline ЭПР точечной цели, м $^{2}$ & 5 \\
\hline Угол наклона базы, градусы & 45 \\
\hline Импульсная мощность, Вт & 180 \\
\hline
\end{tabular}

Полученные данные численных расчетов иллюстрирует рис. 2, на котором показана зависимость дисперсии интерферометрической фазы и ошибки определения высоты от размера интерферометрической базы, для точечной цели и протяженной цели. На графиках вертикальным пунктиром показан предел однозначной оценки интерферометрической фазы в одном пикселе радиолокационного изображения.

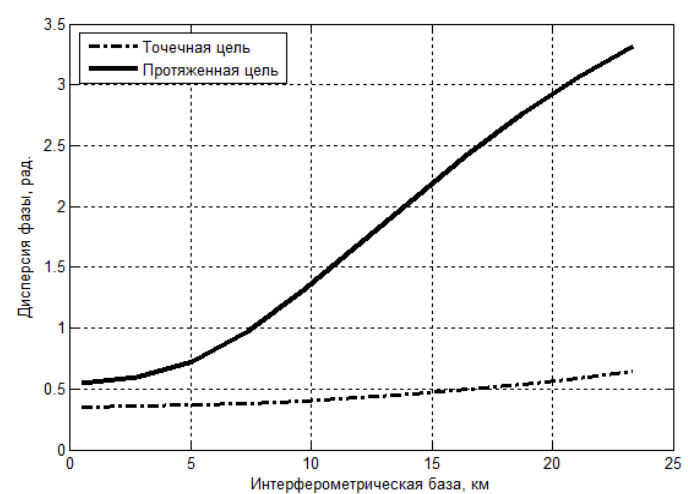

a)

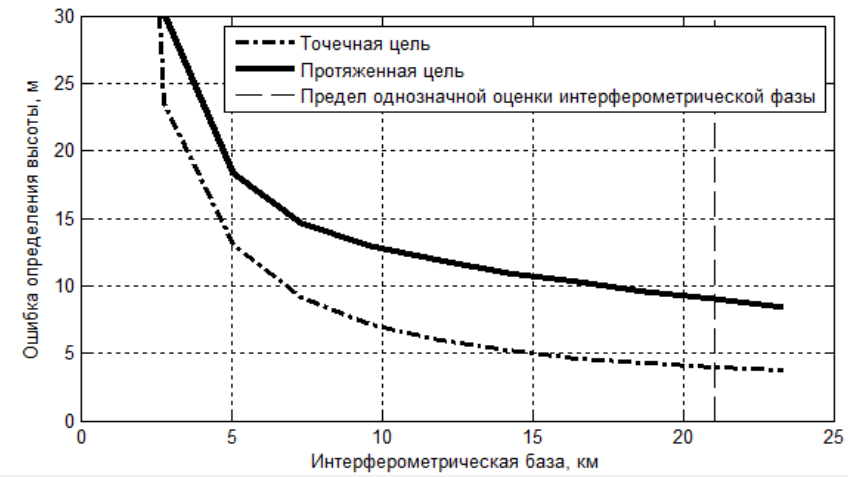

б)

Рис. 2. Зависимости дисперсии фазы (а) и ошибки определения высоты (б) от размера интерферометрической базы, для точечной цели (пунктирная линия) и протяженной цели (сплошная линия), концентрация электронов $N_{e}=10^{12}$, масштаб неоднородностей ионосферы 1000 м

\section{Заключение}

Таким образом, бистатическая схема построения двухпроходной интерферометрической РСА в Р-диапазоне частот, космического базирования, использующая наземный пункт приема, может обеспечить приемлемую точность восстановления высоты точечных целей (до 4 м) и протяженных целей (до 9 м).

\section{References}

[1] Kirilin A.N., Ahmetov R.N., Shahmatov E.V., Tkachenko S.I., Baklanov A.I., Salmin V.V., Semkin N.D., Tkachenko I.S., Goryachkin O.V. Experimental technological small spacecraft "AIST-2D". - Samara: Publishing House of SSC SAM, 2017. - 324 p. 
[2] Goryachkin O.V., Gengurov B.G., Bakeev V.B., Baraboshin A.YU., Nevsky A.V., Skorobogatov E.G. Bistatic P-band SAR for spacecraft «Aist-2D»// Telecommunication Journal, 2015. No. 8. Pp. 34-39.

[3] Goryachkin O.V., Gengurov B.G. Image formation zone in the space bistatic synthetic aperture radar P band // XXII International Scientific and Technical Conference "Radiolocation, Navigation, Communications", Voronezh, 19-21 April 2016.Pp. 1113-1122.

[4] Alyshev Yu. V., Borisenkov A. V., Brainina I. S., Goryachkin O. V., et al. Optimal methods for signal processing in radio engineering and communication systems. - Samara: Publishing house of SSC SAM, 2018. $344 \mathrm{p}$.

[5] Zhu X., Zhang Q., Zhang Y., Dong Z. Analysis of background ionospheric effects on low frequency repeatpass InSAR system // Radar Conference 2015.

[6] Chen A., Zebker H. Reducing Ionospheric Effects in InSAR Data Using Accurate Coregistration // IEEE Transactions on Geoscience and Remote Sensing V. 52, 2014, P.50 - 60.

[7] D.D. Klovsky Transmission of discrete messages via radio. 2 nd ed., M .: Radio and communication, 1982. $304 \mathrm{p}$. 